OPEN ACCESS

Citation: Sandra Francini (2021) Gianfranco Bandini, Carmen Betti, Annalisa Massari (a cura di), Progettare il cambiamento educativo. L'impegno di Marcello Trentanove tra comunità e territorio. Rivista di Storia dell'Educazione 8(1): 81-84. doi: 10.36253/rse-11279

Received: May 31, 2021

Accepted: May 31, 2021

Published: July 5, 2021

Copyright: (c)2021 Sandra Francini. This is an open access, peer-reviewed article published by Firenze University Press (http://www.fupress.com/rse) and distributed under the terms of the Creative Commons Attribution License, which permits unrestricted use, distribution, and reproduction in any medium, provided the original author and source are credited.

Data Availability Statement: All relevant data are within the paper and its Supporting Information files.

Competing Interests: The Author(s) declare(s) no conflict of interest.

Editor: Pietro Causarano, Università di Firenze.
Recensione

\section{Gianfranco Bandini, Carmen Betti, Annalisa Massari (a cura di), Progettare il cambiamento educativo. L'impegno di Marcello Trentanove tra comunità e territorio. Atti del Convegno del 9-10 novembre 2018. Bagno a Ripoli}

\author{
Roma, Anicia Editore, 2020, pp. 183.
}

\author{
SANDRA FranCini \\ Università di Firenze \\ E-mail: safrancini@gmail.com
}

Il titolo del volume che raccoglie gli atti del convegno tenuto a Bagno a Ripoli nel novembre 2018 per ricordare la figura e l'opera di Marcello Trentanove, include nel sottotitolo una parola importante: impegno. Dell'impegno il direttore didattico Trentanove non ne fece economia, consapevole che l'azione, se guidata da un'etica professionale e umana, rappresenta il motorecardine delle trasformazioni sociali. Anche per questo richiamo alla concretezza dell'azione, il testo che qui presentiamo risulta avere un valore in più. I tre curatori - anche testimoni dell'opera formativa messa in atto a Bagno a Ripoli - hanno dato voce a insegnanti, amministratori comunali, rappresentanti dei CEMEA e dell'MCE e a magistrati, che con grande partecipazione ricostruiscono l'esperienza di Trentanove in qualità di direttore nel Circolo di Bagno a Ripoli fra il 1962 e il 1990, anno del suo pensionamento.

Stima e rispetto per questo «direttore pedagogico, presente, esigente, a volte inflessibile» come lo descrive Manola Pignotti, mentre ce ne parla con riconoscenza e affetto (p.28), trovano conferma fin dalle prime pagine del volume attraverso una pluralità di figure che interagirono con lui in quegli anni di scuola. Gli autori ricordano le idee e le scelte organizzative e didattiche che lui propose e difese con forza, sottolineando come quella gestione così innovativa appassionò consapevolmente la cittadinanza che si mosse a suo sostegno facendo sì che l'"utopia trentanoviana" si tramutasse ben presto in un reale, seppur circoscritto, cambiamento. E proprio al cambiamento mirava l'agire di «Marcello Trentanove [che] definiva la scuola pubblica come un'impresa comune fra tutti coloro che vi operano, che vi lavorano e che la utilizzano» (p. 9). La visione di società come "comunità educante", lo portò a considerare tutti gli "attori" dentro la scuola e fuori di essa, dagli insegnanti agli amministratori, dal personale della mensa agli autisti dei 
pulmini, ugualmente centrali per la formazione dei suoi scolari. Questo concetto, che riaffermerà spesso nei suoi interventi, viene ben sottolineato da Vasco Tacconi che evidenzia la concreta collaborazione dell'amministrazione comunale e della comunità locale in quel programma d'avanguardia ricordando che «nulla fu facile, tutto richiese impegno, studio, passione e costanza. Però era bello, perché il confronto - e anche lo scontro - si concentrava sui fatti e non sulle parole» (p.58). La larga adesione al progetto di Marcello viene testimoniata anche da Giuseppe Carrai, Luciano Bartolini, Paola Campani e altri relatori che, con ruoli diversi, condivisero quell'esperienza che rivoluzionò non solo la scuola elementare del comprensorio ripolese, ma dette vita - non appena entrò in vigore nel 1969 la legge istitutiva della scuola materna statale - a sezioni dedicate all'infanzia. Trentanove la considerava un servizio sociale, oltre che educativo, importante (si ricorda che fino ad allora le uniche strutture per l'infanzia erano gli asili gestiti dalle suore), alla pari di ogni altro ordine di scuola al fine di garantire quella "continuità formativa" della persona che lui rese concreta, promuovendo, nella pratica, una diversa organizzazione dell'insegnamento centrata sulla programmazione, la collaborazione e l'alternanza dei docenti nei contesti educativi delle due scuole, materna e elementare.

Nelle pagine introduttive, Annalisa Massari ricorda come «la scuola di Bagno a Ripoli continui tuttora a perseguire le indicazioni didattico-educative che hanno impostato il rapporto con la comunità che si identifica con le sue proposte» (p. 18), mentre riconosce che la realtà nazionale è molto cambiata rispetto agli anni Settanta, anche all'interno delle scuole, e per insegnanti e dirigenti non sempre è possibile dialogare con serenità con i genitori, presi dai tanti problemi dell'oggi.

Rimandando ai bei contributi del libro che ci mostrano più da vicino come, non senza difficoltà e ostacoli, si realizzò l'avventura pedagogica di Trentanove, ci limiteremo qui a ricordare alcune delle sue più note iniziative messe in essere con lucidità e tenace impegno e che, a ben vedere, sono riconducibili ai principi costituzionali che lo accompagnarono nel corso della sua vita e lo guidarono nel suo divenire come cittadino e come uomo di scuola. L'abolizione delle pluriclassi, l'istituzione del tempo pieno (avviato nel 1971), l'aggiornamento continuo degli insegnanti, la creazione della scuola materna e dei nidi per l'infanzia furono fra le sue grandi conquiste. Ma altrettanto rilevante a livello nazionale e internazionale fu l'inserimento, fin dagli anni Settanta, dei bambini disabili nelle classi a tempo pieno, anticipando di gran lunga la legge del 1977 che aboliva di fatto le classi speciali o differenziali.
Già da questo sintetico elenco si può ben capire quale fosse l'idea e il fine della scuola di Marcello: la "sua scuola" doveva essere pubblica e laica, aperta a tutti e a tutte le posizioni ideali, scevra da ogni ideologia, e avere per fine la formazione individuale e sociale, cioè formare il carattere, ritenuto condizione essenziale per una consapevole partecipazione del cittadino alla vita della comunità e dello Stato.

Gli interventi qui raccolti prendono avvio da una realtà toscana del secondo Novecento, mostrandoci come il comprensorio di Bagno a Ripoli si rivelasse all'epoca un'isola educativa virtuosa a fronte della condizione scolastica di moltissime parti della Nazione dove niente era cambiato rispetto al primo dopoguerra: istruzione impartita tramite programmi didattici impigliati in norme burocratiche e in rigidi metodi di insegnamento erano le caratteristiche più evidenti della nostra scuola nel momento in cui le classi popolari, povere di tutto e la cui sola ricchezza possibile era "sapere di più", individuavano nell'accesso all'istruzione l'unica via per ribaltare il corso della loro esistenza. Di tali bisogni, come ben riporta Carmen Betti nel suo intervento, si fecero portavoce i partiti della sinistra (PSI e PCI) che si batterono affinché questi diritti si tramutassero in reali riforme. Dal riferimento storiografico più generale, Betti sposta l'attenzione sull'esperienza educativa messa in atto da Trentanove, ponendosi un punto di domanda, ovvero se quella gestione didattica così avanzata «sia da spiegare come riflesso del clima che ha preparato il '68, momento storico cruciale per le trasformazioni scolastiche, o se non sia stato piuttosto espressione di un itinerario personale in progress, avviatosi molto prima» (p. 60). E qui veniamo alla forte influenza del contesto, dato sempre incidente nella disciplina storica e, ancor più, nella storia di ogni essere umano e Betti lo scandaglia cercando una risposta al suo interrogativo. Lo sguardo da questa angolatura ci mostra, infatti, quanto siano stati influenti l'ambiente, i fatti e le persone, le esperienze vissute fin da ragazzo, nel forgiare e indirizzare le scelte di questo giovane direttore didattico che arriva a Bagno a Ripoli quando ha appena 38 anni portando con sé valori politico-pedagogici forti, uniti a una preesistente idea di scuola alternativa che presto metterà a frutto con intelligenza e determinazione. L'analisi di Betti continua ricordando la militanza di Trentanove nel Partito d'Azione con il quale collabora durante la battaglia per la liberazione di Firenze del '44, esperienza che fu per lui un'importante palestra di formazione civile e politica e segnerà non poco le sue scelte future. La collaborazione e l'amicizia con tanti compagni dei movimenti politici, a cominciare dalla brillante figura di Tristano Codignola, con cui condivise la vita politica dal Partito d'Azione a Unità 
Popolare al Partito Socialista Italiano, come il sodalizio con altre figure di notevoli intellettuali come Margherita Fasolo, Aldo Pettini, Aldo Capitini, Bruno Ciari, furono fondamentali per la sua cultura democratica e la sua capacità organizzativa. Ma come si ricorda in più interventi, e come dirà lui stesso in una testimonianza riportata in appendice al volume, l'esperienza fatta con i CEMEA per oltre trent'anni, dal '51 all'82, rappresentò uno dei "casi" più significativi della sua vita,

perché questa esperienza mi permise di aprire il mio orizzonte ai problemi concreti dell'apprendimento: il fare, il progettare, il vivere insieme, il riflettere, il valutare la corrispondenza dei mezzi con i fini; in una parola, considerare il bambino capace di costruire la propria formazione se posto in una situazione propizia. E quel tirocinio continuo che furono gli stage mi portò a capire gli strumenti e gli atteggiamenti più giusti per "trattare" con gli adulti (p.159).

Dello stile di Trentanove nel trattare con gli adulti ne parla Rosaria Di Santo che, in apertura del suo intervento, ricorda uno dei tratti più significativi di Marcello, cioè l'esserci, l'avere cura di ogni progetto, la presenza vigile ed attenta che infondeva forza in chi sosteneva l'impegno e lo sforzo dell'innovazione educativa (p. 89). Nel concordare con altri relatori, come il suo imprintig didattico, gestionale e relazionale, derivasse in gran parte dalla militanza nelle istituzioni di "educazione attiva", Di Santo ripercorre gli anni che lo videro alla direzione del Circolo didattico di Bagno a Ripoli e ne evidenzia i caratteri distintivi del suo agire come la laicità intesa in più accezioni: la lotta per la scuola pubblica contro le ingerenze ecclesiastiche, la pratica consueta di non dare niente per scontato, il considerare punti di vista diversi anche se ritenuti sbagliati e non pensare di avere sempre ragione. E ancora, se intendiamo combattere posizioni che non condividiamo, dobbiamo prima conoscerle perché nella conoscenza si possono trovare aspetti che non avevamo considerato. Con queste convinzioni sorte dall'intreccio tra ideologia e reale, Trentanove diresse il Circolo di Bagno a Ripoli, persuaso che il cambiamento si dovesse attivare partendo dal "basso", senza aspettarsi riforme dall'alto e, convinto che gli organi ministeriali avrebbero ostacolato ogni rinnovamento, individuò in una "rinnovata figura di insegnante" l'elemento centrale per una scuola diversa nei metodi e nella didattica, come ben riporta Di Santo che, a conclusione del suo lavoro, sottolinea quanto sarebbe importante che i futuri insegnanti di Scienze della formazione primaria conoscessero questa esperienza per meglio orientarsi nell'attività quotidiana e in quella di lungo periodo.

Ma ancora oggi si presta poco ascolto alla richie- sta di una "scuola rinnovata" e poco se ne parla nella discussione politica come sottolinea Gianfranco Bandini. Da studioso di tematiche pedagogiche egli rileva una persistente difficoltà a migliorarne il funzionamento e a individuarne con chiarezza i fini. Partendo dalle biografie raccolte nel bel libro Maestri nell'ombra (2011), di cui lui stesso è stato curatore insieme a Caterina Benelli, rileva la costante presenza di elementi comuni a questi "maestri dimenticati" che tanto hanno dato alla scuola, dei quali Marcello Trentanove è un grande esempio. Bandini ne sottolinea la competenza, la capacità di cogliere in anticipo le difficoltà dei contesti educativi, la prontezza nel trovare soluzioni, la passione per il suo lavoro. Il suo articolato intervento mostra come le proposte veicolate dalle idee liberiste, a cavallo tra il XX il XXI secolo, e condivise da rilevanti sponde politiche, abbiano danneggiato la nostra scuola proponendo modelli gestionali competitivi, più vicini alla conduzione di un'azienda che a una istituzione-cardine come la scuola a cui si dovrebbe assegnare l'importante compito di educare al confronto, all'uguaglianza delle opportunità, alla tutela delle differenze. Trentanove lavorò sempre perché questi valori fossero alla base dell'azione educativa, come ben illustra Beniamino Deidda, definendolo un pratico illuminato. La scelta di non essere un teorico della pedagogia o della didattica gli permise di lavorare sempre - e lui aveva l'ansia del fare, ricorda Deidda sulla scorta della sua robusta preparazione al fine di rendere più avanzati i metodi, i contenuti e la didattica nella scuola. Capacità e passione dettero vita a un modello educativo non autoritario, la cui essenza era l'inclusione, l'ascolto e la comprensione dei ragazzi e delle loro famiglie. Ma la battaglia che più lo impegnò sul piano culturale, continua Deidda, fu l'esigenza di una scuola che, come abbiamo visto, voleva solo laica e, contrario alle scuole private, cercò sempre di affermare e di rendere condivisi i valori profondi della nostra Costituzione repubblicana.

L'intervento di Gianfranco Staccioli - che come Trentanove fu membro e dirigente dei CEMEA toscani - ribadisce come Marcello tenesse molto a quella "coerenza ideale" che, fin dall'immediato dopoguerra, lo aveva portato a partecipare a organismi associativi pacifisti e a associazioni e gruppi attinenti sia alla persona che al contesto politico sociale. Fu quindi naturale per lui aderire ai principi base dell'educazione attiva propugnata nei CEMEA di cui Staccioli ricorda le stimolanti, accese e feconde discussioni al suo interno. Un tema molto dibattuto, scrive, riguardava due principi-base del movimento: il principio del rispetto per ogni obiezione o convinzione altrui e il principio del contesto da mantenere accogliente e facilitante per la crescita e la consapevolez- 
za della persona, anche in situazioni meno ideali. Marcello fece proprio il significato profondo di queste parole, insieme al concetto deweyano del qui e ora, i cui dettami incoraggiavano la creatività ma, allo stesso tempo, richiamavano alla responsabilità e al senso della realtà. Nel rievocare le tante iniziative condotte nel movimento, Staccioli ricorda che in esse Marcello era spesso il "motore trainante" come, ad esempio, quando prese avvio, negli anni a partire dal 1963, un proficuo dialogo con i più aggiornati e attivi medici psichiatri fiorentini e italiani (fra cui Franco Basaglia), oltre a pedagogisti e docenti universitari, con l'obiettivo di promuovere metodologie più appropriate nella cura e nell'assistenza dei malati di mente. La testimonianza di Gianfranco Staccioli è coinvolgente nel richiamare alla mente anni densi di progettualità, di principi, di valori, di parole oggi dimenticate, ma che sarebbe importante riportare alla luce. I saggi contenuti nel volume chiudono con le riflessioni di Paolo Borin che approfondisce e descrive con cura l'esperienza del tempo pieno a Bagno a Ripoli, nata, come si è detto, nel 1971. Questa complessa sperimentazione ribaltava i parametri tradizionali dell'insegnamento e della relazione educativa partendo, come abbiamo visto, dalle teorie di Dewey fino alle esperienze dei CEMEA.

Il ricco volume si conclude con la diretta, preziosa testimonianza di Marcello Trentanove: sono documenti diversi, fra cui un'intervista dove egli racconta, in prima persona, la propria storia, ricorda gli amici più cari e, naturalmente, ci parla della sua "avventura didatticoeducativa" vissuta a partire da quel vivace periodo storico rappresentato dagli anni Sessanta, pieno di fiducia e speranza nel futuro.

E oggi, in un momento difficile come quello che il mondo intero sta affrontando, in cui adolescenti e giovani vivono con disagio e preoccupazione la loro stessa esistenza, spesso in ansia per le difficoltà personali e per quelle socio-economiche delle loro famiglie, proprio da questi ragazzi potrebbe arrivare una proposta di aiuto per tutti. Spetta agli adulti sostenere la loro energia, abbandonando la comunicazione confusa che da tempo arriva loro da più parti, a volte addirittura considerati untori da guardare con sospetto, a volte vittime perché lontani dalla scuola, dagli amici o dallo sport di gruppo. Forse ci sarebbe bisogno di responsabilizzarli facendoli partecipi del ruolo che possono svolgere in questi mesi di pandemia grazie alla loro energia, alla loro fantasia, al loro impegno. Spetta in particolare alla politica renderli protagonisti, investendo risorse fisiche, morali e economiche nell'educazione, nell'insegnamento e nella scuola. L'impegno che, come si è visto, ha rappresentato una costante nell'operato di Marcello Trentanove, resta anco- ra una volta il punto centrale per chi vorrà ripensare il "tempo del dopo". 